\title{
A design study for synchrotron-based high-numerical-aperture scanning illuminators
}

\author{
Patrick P. Naulleau and Paul E. Denham \\ Center for X-Ray Optics, Lawrence Berkeley National Laboratory, Berkeley, CA 94720
}

\begin{abstract}
Scanning illumination systems provide for a powerful and flexible means of controlling illumination coherence properties. In these systems, the desired illumination divergence is effectively synthesized through a scanning process. This method has recently been used at extreme ultraviolet (EUV) wavelengths to implement a programmable pupil fill microfield exposure system employed to lithographically characterize a 0.1 -numerical aperture (NA) alphaclass EUV stepper optic. The specifics of the implementation used in that case, however, make it difficult to directly extend the implementation to higher NA optics such as the 0.3-NA MicroExposure Tool (MET) optic currently being developed for advanced lithographic studies. Here we present scanning illuminator configurations suitable for implementing high-NA lithography capabilities at a synchrotron beamline. In particular we consider the application to the 0.3-NA MET optic with a design field of view of $1 \times 3 \mathrm{~mm}$ at the object plane.
\end{abstract}

Keyword: extreme ultraviolet, lithography, microscopy, condenser, synchrotron, coherence 


\section{Introduction}

Microfield static exposure tools [1-3] have and are expected to continue to play a crucial role in the development and commercialization of extreme ultraviolet (EUV) lithography [4]. Although not under serious consideration for manufacturing applications, synchrotron radiation provides a convenient well-characterized debris-free source for such microfield systems. While synchrotron sources are well suited to metrology applications [5], the problem with using these sources for lithography is the poor match between the intrinsic coherence properties of the source and those required of a lithographic tool. Synchrotron radiation is typically much more coherent than one desires for lithographic printing. This is particularly true of undulator radiation [6], but also holds for bend-magnet radiation.

In general, lithography studies most relevant to commercial applications require the illumination coherence area to be only slightly larger than the diffraction-limited resolution of the lithographic optic. The illumination coherence factor $(\sigma)$ is typically chosen to be approximately 0.7 , where $\sigma$ can be described as the ratio of the illumination divergence to the lithographic optic object-side numerical aperture (NA). For critical illumination systems, this translates to the ratio of the condenser NA to the lithographic optic entrance NA. Given that synchrotron sources are typically characterized by low divergence and relatively small spatial extent, achieving useful $\sigma$ values over field sizes even relevant for microfield systems is challenging. A benefit of starting with high coherence, however, is that, in principle, one has the freedom to customize the coherence through the use of active components.

At visible wavelengths, an often-used method for controllably reducing spatial coherence is to scatter the beam with rotating ground glass $[7,8]$. The ground-glass surface can be viewed as the new effective source and the source size changed simply by changing the illuminating beam 
size on the ground glass. This method, however, is presently impractical at EUV wavelengths due to the difficulties involved in obtaining adequately efficient equivalent ground glass structures.

An alternative coherence-control method, more suitable to EUV applications, involves a scanning process [9-10]. Here, the desired illumination divergence is effectively synthesized through the scanning process. This method has recently been used at EUV wavelengths to implement a programmable pupil fill microfield exposure system designed to operate with a 0.1NA lithographic optic [11]. This system, installed at Lawrence Berkeley National Laboratory's Advanced Light Source synchrotron radiation facility, has enabled the early lithographic characterization [3] of an alpha-class EUV stepper optic [12]. The specifics of the implementation used in that case, however, make it difficult to directly extend the implementation to higher NA optics such as the 0.3-NA Micro-Exposure Tool (MET) optic currently being developed for advanced lithographic studies $[13,14]$.

Here we present scanning illuminator configurations suitable for implementing high-NA lithography capabilities at a synchrotron beamline. In particular we consider the application to the 0.3-NA MET optic with a design field of view of $1 \times 3 \mathrm{~mm}$ at the object plane and a target maximum $\sigma$ value of 1 .

\section{Limitations of previous design}

A significant limitation of the previously implemented EUV scanning illuminator (Fig. 1) [11] was the limited speed of the device forcing exposure times to be on the order of 4 seconds long. This constraint was due to mechanical limitations in the two-dimensional tilt table used as the scanner. This speed restriction would become even more severe for the 0.3-NA MET optic of interest here, which would require larger scan angles or significantly increased optical demagnification from the scanner to 
the reticle. Increasing the optical demagnification, however, would come at the unacceptable cost of reduced illumination field size. To overcome the scan-speed issue, it is beneficial to implement a compound scanner comprised of two one-dimensional scanners, of which high-speed large-angle units are readily available commercially.

Another limitation of the previous implementation was the fact that the illumination area was required to be very close to the edge of the reticle due to the use of a single spherical mirror serving as both the condenser and the only fold mirror between the scanner and the reticle. The use of a spherical mirror precludes angles of incidence deviating too far from normal, which would lead to large astigmatism. This limitation can be overcome by a variety of methods including the use of additional fold mirrors, implementing an astigmatic scanner, and using a non-spherical single or compound condenser optic.

\section{Configurations compatible with the MET}

Perhaps the most straightforward extension of the Fig. 1 illuminator concept to the MET is to add two fold mirrors to the system and to implement a compound scanner as described above (Figs. 2). This configuration supports the use of a full 6-inch reticle and is comprised simply of flat scanning and turning mirrors and a single spherical imaging (condenser) mirror. The drawbacks of this system are the optical losses imposed by the numerous multilayer reflections and the complexity of placing and aligning two optics in the tight mechanical space between the MET optic and the reticle. Compared to the prior implementation, the use of three additional mirrors would lead to an optical throughput loss of at least $66 \%$ assuming $70 \%$ reflectivity multilayers.

We note that, in principle, the first turning mirror could be eliminated by moving the rest of the system up and adjusting the geometry of the compound scanner. However, in our particular implementation, the first turning mirror is required for mechanical compatibility with the current 
beamline and endstation [3,11] installed at sector 12 of the Advanced Light Source. Moreover, in the current beamline configuration, that turning mirror is mounted onto a two-dimensional angle-scanning stage (previously used as the coherence-synthesis scanner), which could now conveniently serve as a field scanner, thereby enhancing the illumination uniformity. In this dual-domain scanner configuration one can, in principle, simultaneously synthesize both the source divergence (spatial frequency spectrum) and source size and uniformity.

By eliminating the low astigmatism requirement previously placed on the spherical condenser optic, we can increase its angle of incidence and forego the need for the final turning mirror. This can be accomplished by setting the distance between the $x$ and $y$ scanning elements to coincide with the astigmatic foci determined by the angle of incidence on the spherical mirror. In practice, however, this leads to unacceptably large separations between the $x$ and $y$ scanners. The scanner separation can again be reduced if we allow one of the scanners to contain optical power in the cross-scan direction (i.e. a cylindrical scanning mirror). In this case, the condenser can be viewed as actually being a compound optic where one of the elements serves as both a condenser element and a scanner element.

Figures 3 and 4, respectively, show $y-z$ and $x-z$ plane diagrams of an illuminator of this configuration. The $x$ scanner remains flat and the $y$ scanner becomes a cylinder with power in the $x$ direction. Combined with the spherical mirror, the $y$ scanner re-images the $x$ scanner in the $x$ direction to the reticle. Imaging from the $y$ scanner to the reticle in the $y$ direction is performed simply by the spherical mirror. Increasing the propagation angle from the $y$ scanner to the spherical mirror from 0 degrees to the 26 degrees allows the required scan range from the $x$ scanner to be reduced from $+/-10$ degrees to $+/-3.7$ degrees. This is due to the fact that we are apportioning more optical power to the spherical mirror and effectively shortening the focal length of the $x$-direction imaging compound optic. The upper limit on this angle is set by obscuration constraints determined by the mask size and 
the desired reticle stage travel. We note that the required $y$ scanner angle range in this configuration is approximately $+/-0.75$ degrees.

\section{Stationarity}

To first order, the field size is simply determined by the illumination size on the scanning mirrors combined with the optical demagnification introduced by the condenser optic(s). However, we have not yet addressed the stationarity of the illumination. As evidenced in Fig. 2, a constraint imposed by the shallow tilt angle of the mask ( 4 degrees), is that achieving $\sigma=1$ illumination leaves virtually no mechanical clearance between the final illuminator optic and the light entering the imaging optic in the $y$ direction. We note that this same constraint does not exist in the $x$ direction. The limited clearance raises the issue of vignetting for source points displaced from the axis in the $y$ direction. Ideally, to eliminate vignetting one would like the center of the synthesized emission cone from all source points to strike the center of the spherical lens. Because, for the nominal implementation presented here, the virtual source size in the $y$ direction is approximately equal to one-half the spherical mirror size required to support a $\sigma$ of 1 , and the central rays can be assumed to be roughly collimated (incoming illumination from the beamline is approximately collimated), any $\sigma$ value of greater than 0.5 will suffer from spatial variance across the field. This issue can be addressed by the use of a field lens, which, in practice, means adding $y$ curvature to the $y$ scanner, making it a toroid. However, even with this correction, strictly speaking the system still suffers from spatial variance in terms of pupil-fill shift. With the $y$ scanner $y$ focal length set to match the distance to the spherical mirror, the fractional pupil-fill shift across the field can be shown to be 0.067 , which is arguably an acceptably small value. 
The situation, however, is worse for $x$ where we have a larger field size ( $3 \mathrm{~mm})$. In this case the fractional pupil-fill shift is 0.2 , which is unacceptably large. The situation becomes more tolerable as the field size is reduced to $1.5 \mathrm{~mm}$ or below. We note these $x$-pupil-fill-shift considerations are based on the central ray of the emission cone striking the center of the spherical mirror. However, as described above for $y$, this cannot occur without the use of an $x$ field lens. Without this field lens, the shift is considerably worse. Designing the field lens to minimize pupil shift instead of striking the center of spherical lens, enables the shift to be virtually eliminated even over the 3-mm field size. Achieving this without vignetting, however, requires the spherical lens to be larger in $x$ than it is in $y$ (recall that the $y$ size of the lens is limited by beam clearance constraints.) The required $\mathrm{x}$-direction size increase is approximately 3 $\mathrm{mm}$ on a baseline diameter of approximately $13 \mathrm{~mm}$.

Part of the motivation for utilizing a cylindrical $y$ scanner as described above was to avoid complications in procuring a toroidal optic to replace the final spherical optic. The vignetting considerations above, however, lead to a requirement for a toroidal $y$ scanner. Thus, there is no reason to eliminate the use of a toroid in place of the sphere as the final condenser optic. This allows the $y$ scanner to again become a simple cylinder in $y$. This stationary toroidal condenser configuration is shown in Fig. 5.

The $x$ scanner is a concave cylinder in $x$ and should have a $x$ diameter of approximately 30 $\mathrm{mm}$. The required scan range for this mirror is $+/-0.5$ degrees. The optical distance from the $x$ scanner to the $y$ scanner is $100 \mathrm{~mm}$ and the $y$ scanner is a concave cylinder in $y$. The $y$ scanner should have a $y$ diameter of at least $10 \mathrm{~mm}$ and a $x$ diameter of at least $32 \mathrm{~mm}$ to support the required NA in the $x$ direction. The required scan range for the $y$ mirror is also $+/-0.5$ degrees. The optical distance from the $y$ scanner to the spherical mirror is $830 \mathrm{~mm}$ at an angle of 26 
degrees from the $x-z$ plane. The toroidal mirror has a $y$ radius of curvature of $220 \mathrm{~mm}$ and a $x$ radius of curvature of $174 \mathrm{~mm}$.

\section{Discussion}

In conclusion, extending the previously described scanning EUV illuminator concept [11] to use with higher-NA optics and extended field sizes ideally requires the use of a toroidal condenser mirror as well as cylindrical scanning mirrors. The implementation of a compound scanner comprised of two one-dimensional scanners enables a scanning speed increase of approximately a factor of 6 compared to the previously described single-element two-dimensional scanner [11]. The one-dimensional scanners can be implemented using flexure suspension galvanometers [17], which can readily be made vacuum compatible. Using these devices, we have achieved non-harmonic scan rates of $500 \mathrm{~Hz}$ using the worst-case mirror ( $x$ scanner) from the design shown in Fig. 5 (the previous two-dimensional implementation was limited to a $80 \mathrm{~Hz}$ scan rate). Using the non-harmonic configuration facilitates the generation of arbitrary patterns, however, if circular fill patterns are exclusively used, operating with harmonic devices can further increase the scan rate. Additionally, because there is a direct tradeoff between maximum scan speed and scanning mirror weight and size, scanning rates could also be increased by shrinking the field of view. This is due to the fact that the scanning mirrors themselves are re-imaged to the reticle, thus the field size is directly proportional to the scanning mirror size. Finally, because this scanning system is expected to be significantly more accurate than that used in the previous implementation, averaging of multiple pupil scans [11] may not be required providing another factor of 4 potential scan time decrease.

Although the illuminator designs described here all involved near-normal incidence multilayer coated optics, similar designs can be implemented with grazing incidence optics. We note that this would not be the case for the single-element two-dimensional scanner (Fig. 1) as deflection 
orthogonal to the plane of incidence is highly insensitive to mirror tilts. A potential advantage of grazing incidence optics is the increased reflectivity compared to multilayer optics (grazing incidence reflectivity at EUV is typically $80 \%$ compared to $70 \%$ for the ideal multilayer reflectivity). Additionally, a complete grazing-incidence implementation would enable the system to be used over a significantly larger wavelength range, which although not of concern for lithography applications could be important for other applications such as soft x-ray microscopy.

This research was performed at Lawrence Berkeley National Laboratory and supported by International Sematech and the Director, Office of Science, Office of Basic Energy Science, of the US Department of Energy under contract No. DE-AC03-76SF00098.

\section{References}

1. J. Goldsmith, K. Berger, D. Bozman, G. Cardinale, D. Folk, C. Henderson, D. O'Connell, A. Ray-Chaudhuri, K Stewart, D. Tichenor, H. Chapman, R. Gaughan, R. Hudyma, C. Montcalm, E. Spiller, J. Taylor, J. Williams, K. Goldberg, E. Gullikson, P. Naulleau, J. Cobb, "Sub-100-nm lithographic imaging with an EUV 10× microstepper," Proc. SPIE Vol. 3676, 264-271 (1999).

2. K. Hamamoto, T. Watanabe, H. Tsubakino, H. Kinoshita, T. Shoki, M. Hosoya, "Fine pattern replication by EUV lithography," Journal of Photopolymer Science \& Technology 14, 567$572(2001)$.

3. P. Naulleau, K. Goldberg, E. Anderson, D. Attwood, P. Batson, J. Bokor, P. Denham, E. Gullikson, B. Harteneck, B. Hoef, K. Jackson, D. Olynick, S. Rekawa, F. Salmassi, K. Blaedel, H. Chapman, L. Hale, P. Mirkarimi, R. Soufli, E. Spiller, D. Sweeney, J. Taylor, C. Walton, D. O'Connell, R. Stulen, D. Tichenor, C. Gwyn, P. Yan and G. Zhang, “Sub-70-nm 
EUV Lithography at the Advanced Light Source Static Microfield Exposure Station Using the ETS Set-2 Optic,” J. Vac. Sci. \& Technol. B, to be published (2002).

4. R. Stulen and D. Sweeney, "Extreme ultraviolet lithography," IEEE J. Quantum Electron. 35, 694-699 (1999).

5. D. Attwood, G. Sommargren, R. Beguiristain, K. Nguyen, J. Bokor, N. Ceglio, K. Jackson, M. Koike, and J. Underwood, "Undulator radiation for at-wavelength interferometry of optics for extreme-ultraviolet lithography," Appl. Opt. 32, 7022-7031 (1993).

6. C. Chang, P. Naulleau, E. Anderson, and D. Attwood, "Spatial coherence characterization of undulator radiation," Opt. Comm. 182, 24-34 (2000).

7. M. V. R. K. Murty, "Interference between wave fronts rotated or reversed with respect to each other and its relation to spatial coherence,” J. Opt. Soc. Am. 54, 1187-1190 (1964).

8. W. Martienssen and E. Spiller, "Coherence and Fluctuations in Light Beams," Am. J. Phys. 32, 919-926 (1964).

9. K. Itoh and Y. Ohtsuka, "Illumination with a moving light source," Opt. Comm. 31, 119-124 (1979).

10. K. Itoh and Y. Ohtsuka, “Coherence control by laser scanning,” Appl. Opt. 19, 3184-3188 (1980).

11. P. Naulleau, K. Goldberg, P. Batson, J. Bokor, P. Denham, and S. Rekawa, “A Fouriersynthesis custom-coherence illuminator for EUV microfield lithography," to be published, Appl. Opt. (2003).

12. D. W. Sweeney, R. Hudyma, H. N. Chapman, and D. Shafer, "EUV optical design for a 100 nm CD imaging system," in Emerging Lithographic Technologies II, Y. Vladimirsky, ed., Proc. SPIE 3331, 2-10 (1998). 
13. J. Taylor, D. Sweeney, R. Hudyma, L. Hale, T. Decker, G. Kubiak, W. Sweatt, N. Wester, "EUV Microexposure Tool (MET) for near-term development using a high NA projection system,” 2nd International EUVL Workshop October 19-20, 2000 (http://www.sematech.org/public/resources/litho/).

14. R. Hudyma, J. Taylor, D. Sweeney, L. Hale, W. Sweatt, N. Wester, "E-D characteristics and aberration sensitivity of the Microexposure Tool (MET)," 2nd International EUVL Workshop October 19-20, 2000 (http://www.sematech.org/public/resources/litho/).

15. P. Naulleau, K. Goldberg, E. Anderson, P. Batson, P. Denham, S. Rekawa, and J, Bokor, “At wavelength characterization of the Engineering Test Stand Set-2 optic,” J. Vac. Sci. \& Technol. B 19, 2396-2400 (2001).

16. D. Tichenor, A. Ray-Chaudhuri, S. Lee, H. Chapman, W. Replogle, K. Berger, R. Stulen, G. Kubiak, L. Klebanoff, J. Wronosky, D. O’Connell, A. Leung, K. Jefferson, W. Ballard, L. Hale, K. Blaedel, J. Taylor, J. Folta, E. Spiller, R. Soufli, G. Sommargren, D. Sweeney, P. Naulleau, K. Goldberg, E. Gullikson, J. Bokor, D. Attwood, U. Mickan, R. Hanzen, E. Panning, P. Yan, J. Bjorkholm, and C. Gwyn, "Initial Results from the EUV Engineering Test Stand," Proc. SPIE Vol. 4506, 639-645 (2001).

17. The flexure suspension galvanometers were manufactured by Nutfield Technology, Inc., 49 Range Road, Windham, NH 03087. 


\section{List of Figures}

Fig. 1. Prior configuration [11] of the programmable coherence synchrotron-based scanning illuminator.

Fig. 2. Extension of the Fig. 1 illuminator concept to the MET by adding two fold mirrors to the system and implementing a compound scanner. This configuration supports the use of a full 6-inch reticle and is comprised simply of flat scanning and turning mirrors and a single spherical imaging (condenser) mirror.

Fig. 3. $y-z$ plane diagram of a compound condenser illuminator, where the $y$ scanning element also serves as an $x$-direction condenser element. The $x$ scanner remains flat and the $y$ scanner is a cylinder. Combined with the spherical mirror, the $y$ scanner re-images the $x$ scanner in the $x$ direction to the reticle. Imaging from the $y$ scanner to the reticle in the $y$ direction is performed simply by the spherical mirror.

Fig. 4. $x$ - $z$ plane diagram of illuminator in Fig. 3.

Fig. 5. Single element toroidal condenser design with field lenses providing stationary illumination across the field of view. 


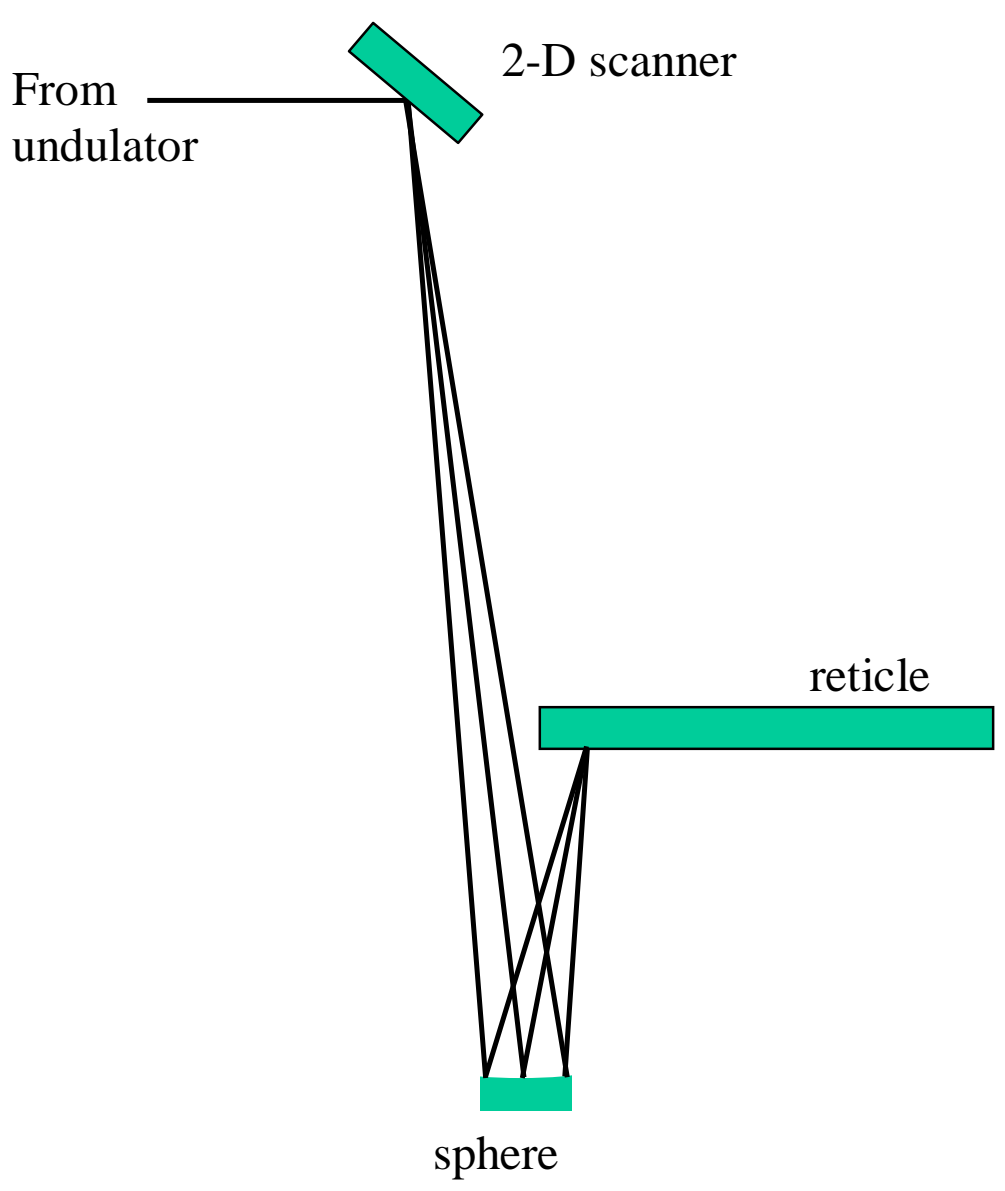

Fig. 1. Prior configuration [11] of the programmable coherence synchrotron-based scanning illuminator. 


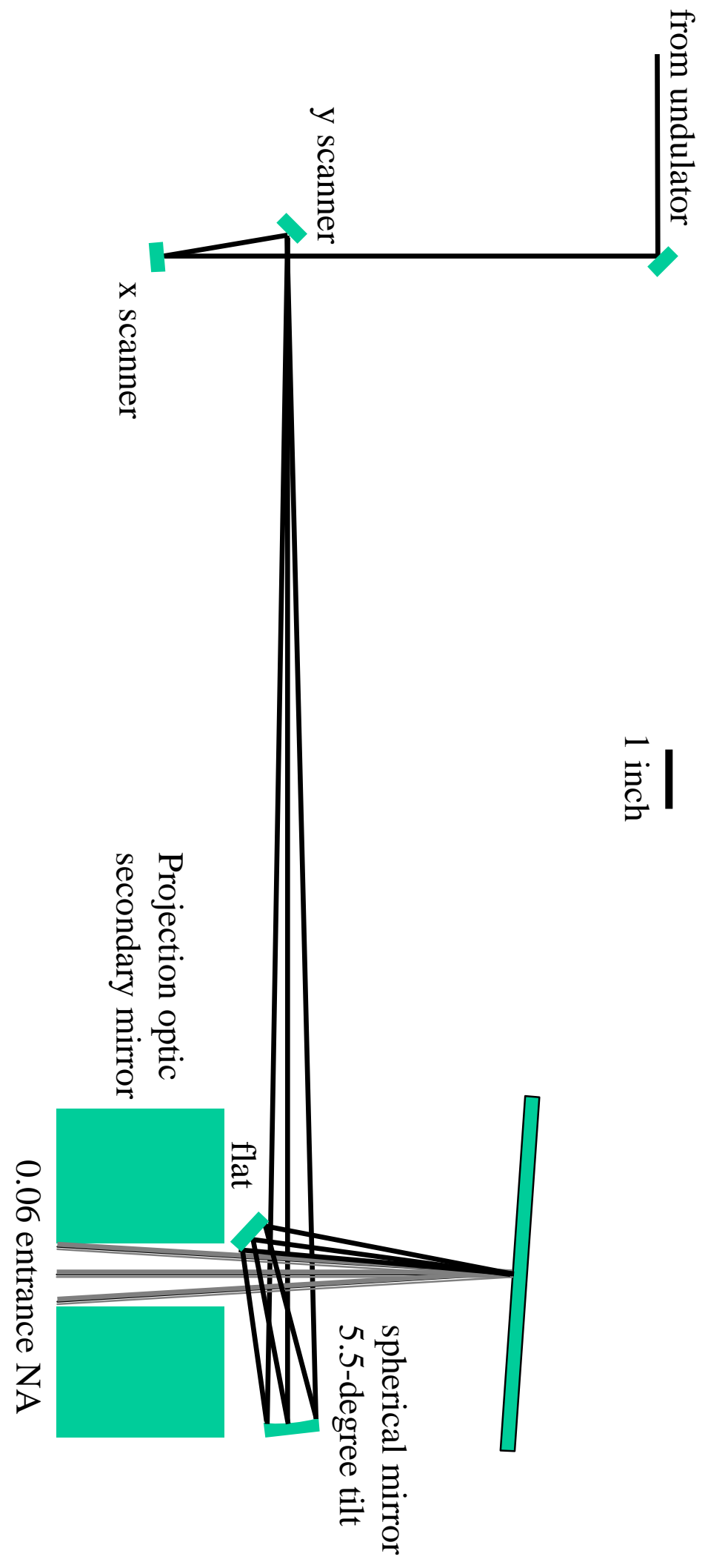

Fig. 2. Extension of the Fig. 1 illuminator concept to the MET by adding two fold mirrors to the system and implementing a compound scanner. This configuration supports the use of a full 6-inch reticle and is comprised simply of flat scanning and turning mirrors and a single spherical imaging (condenser) mirror. 


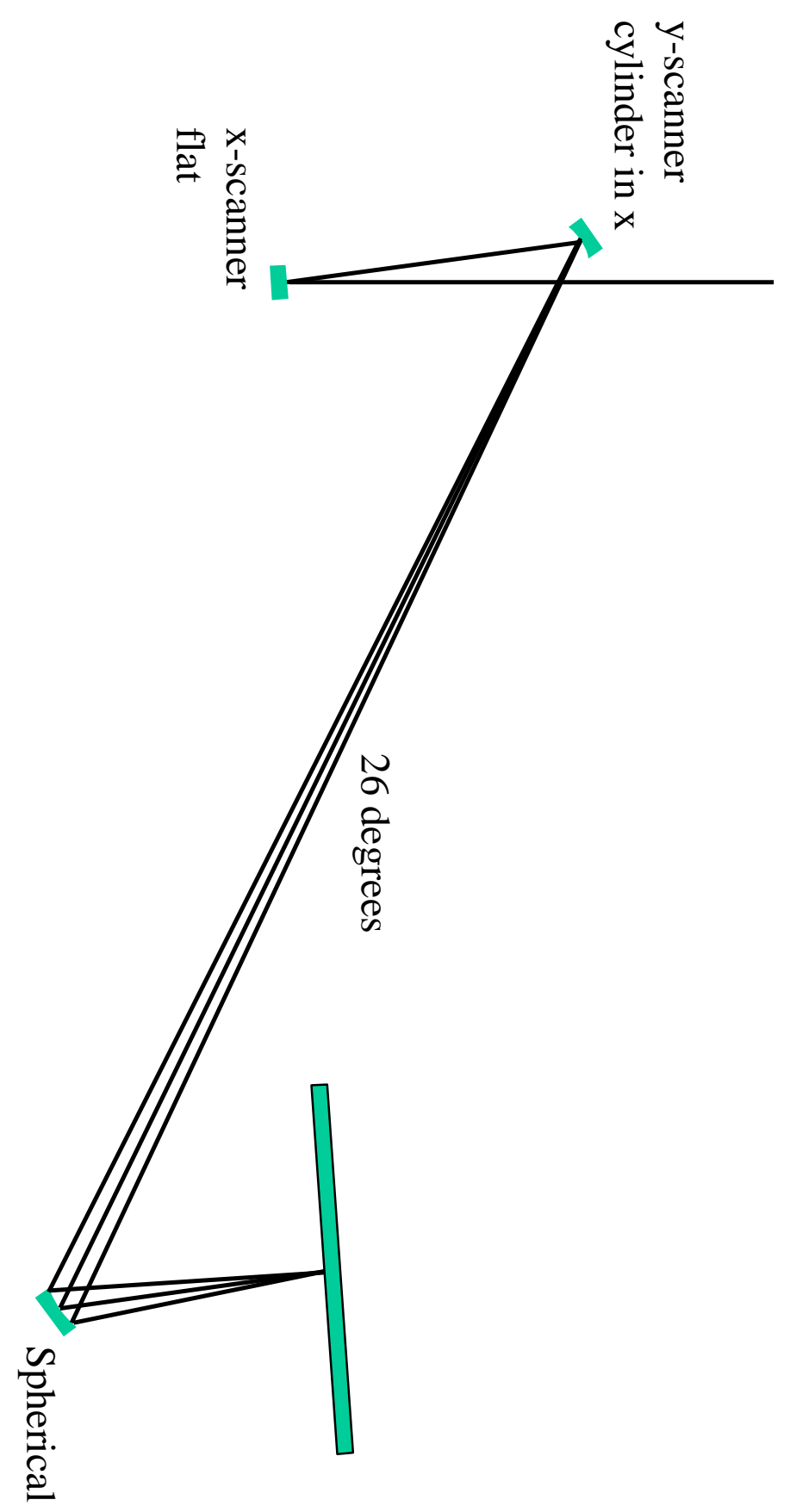

Fig. 3. $y-z$ plane diagram of a compound condenser illuminator, where the $y$ scanning element also serves as an $x$-direction condenser element. The $x$ scanner remains flat and the $y$ scanner is a cylinder. Combined with the spherical mirror, the $y$ scanner re-images the $x$ scanner in the $x$ direction to the reticle. Imaging from the $y$ scanner to the reticle in the $y$ direction is performed simply by the spherical mirror. 


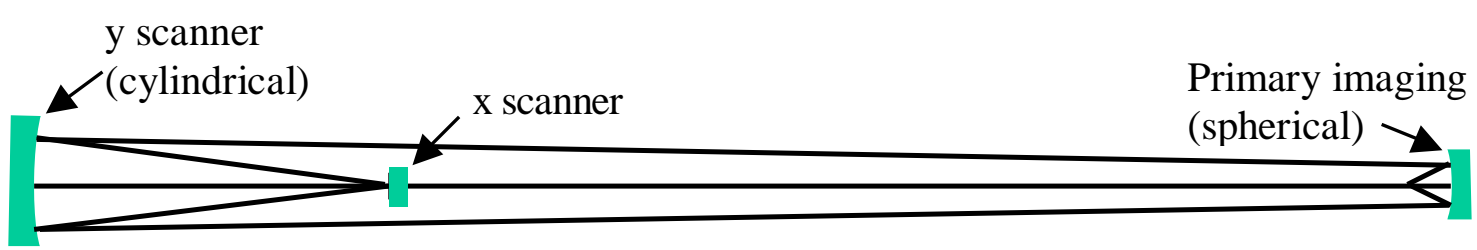

Fig. 4. $x-z$ plane diagram of illuminator in Fig. 3. 


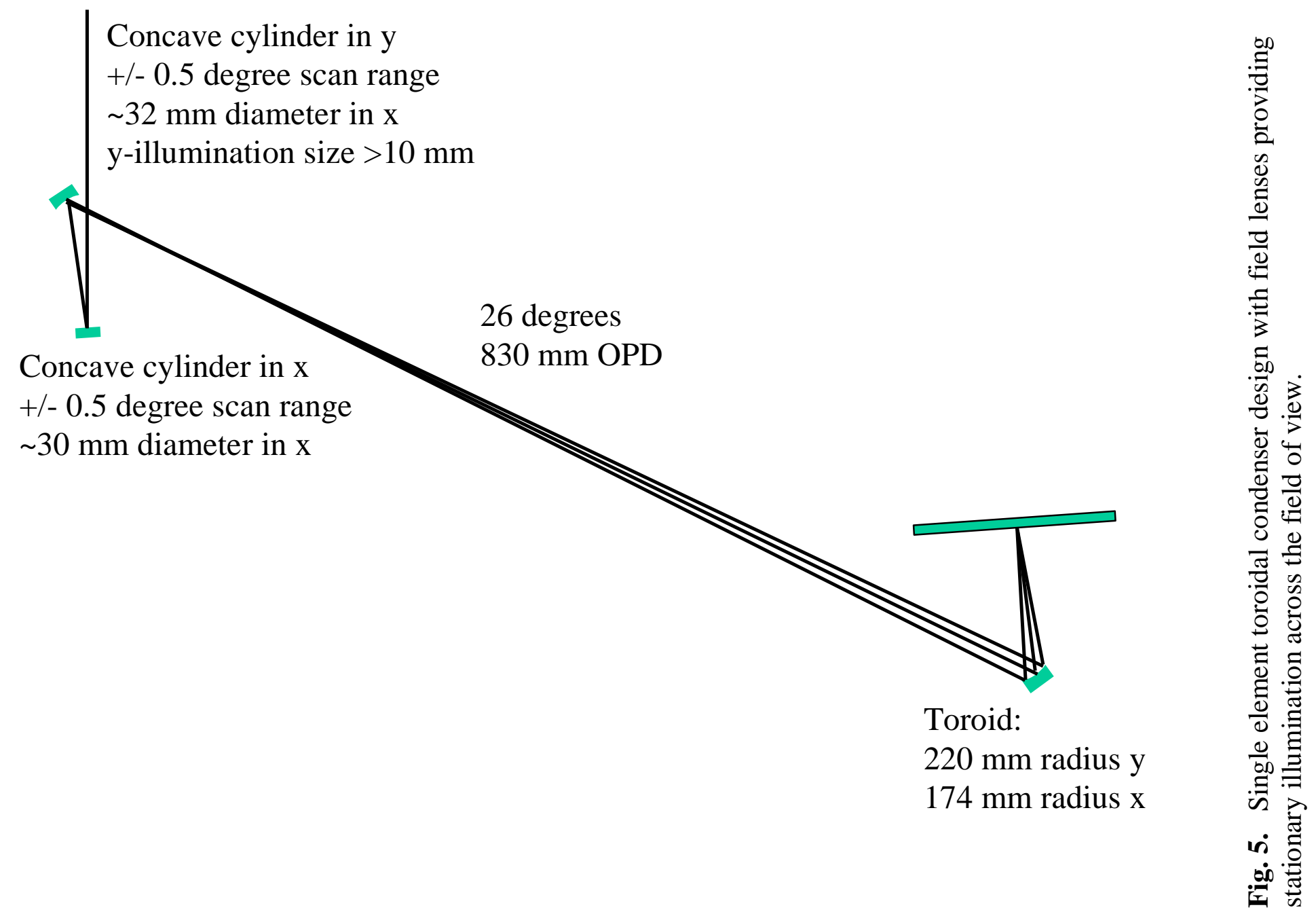

